\title{
THE SYMMETRIC SEMI-CLASSICAL ORTHOGONAL POLYNOMIALS OF CLASS TWO AND SOME OF THEIR EXTENSIONS
}

\author{
G. FILIPUK AND M.N. REBOCHO
}

\begin{abstract}
We study symmetric sequences of orthogonal polynomials of class two related to Stieltjes functions satisfying a Riccati type differential equation with polynomial coefficients. We show difference equations for the recurrence coefficients of the orthogonal polynomials as well as for related quantities. Some of such recurrences are identified with discrete Painlevé equations.
\end{abstract}

KEYwords: Orthogonal polynomials; Semi-classical class; Freud weights; LaguerreFreud equations; Painlevé equations; Stieltjes function.

Math. Subject Classification (2000): 33C45, 33C47, 42C05.

\section{Motivation and preliminary results}

Symmetric orthogonal polynomials on the real line, $\left\{P_{n}(x)=x^{n}+\ldots\right\}_{n \geq 0}$, are characterized in terms of the three term recurrence relation

$$
P_{n+1}(x)=x P_{n}(x)-\gamma_{n} P_{n-1}(x), \quad n=0,1, \ldots,
$$

with $P_{-1}(x)=0, P_{0}(x)=1$. The parameters $\gamma_{n}$, known as recurrence relation coefficients, satisfy $\gamma_{n} \neq 0, n \geq 1$. Integrating with respect to the orthogonality measure, $\mu$, gives us the representation

$$
\gamma_{n}=\frac{1}{h_{n}} \int_{I} x P_{n} P_{n-1}(x) d \mu(x), \quad h_{n}=\int_{I} P_{n-1}^{2}(x) d \mu(x), \quad n \geq 1 .
$$

Here, $I$ is the support of $\mu$.

A very well-known class of symmetric orthogonal polynomials is the one related to semi-classical weights, characterized through a differential equation

\footnotetext{
Received June 22, 2018.

The authors thank CMUC (Centre for Mathematics of the University of Coimbra) for hospitality. G.F. acknowledges the support of the National Science Center (Poland) vis grant OPUS 2017/25/B/BST1/00931. The partial support of G.F. by the Alexander von Humboldt Foundation is acknowledged. The work of M.N.R. was partially supported by the Centre for Mathematics of the University of Coimbra - UID/MAT/00324/2013, funded by the Portuguese Government through FCT/MCTES and co-funded by the European Regional Development Fund through the Partnership Agreement PT2020.
} 
known as Pearson equation [19],

$$
\frac{1}{w(x)} \frac{d}{d x} w(x)=\frac{C(x)}{A(x)}
$$

with the property of symmetry in $w$. A systematic study of symmetric semiclassical weights and the corresponding orthogonal polynomials, began with G. Freud in the 1970's (see [18]). Weights of the form

$$
w(x)=\exp (-Q(x))
$$

with $Q$ an even, non-negative and continuous real valued function defined on the real line (satisfying certain conditions involving its first and second derivatives), are nowadays commonly known as Freud-type weights. The cases $Q(x)=|x|^{m}, m \in \mathbb{N}$, have been extensively studied, main references and results can be found in the introduction section of [11]. A common topic of research concerns the derivation and study of the systems of nonlinear difference equations satisfied by the recurrence relation coefficients of the corresponding orthogonal polynomials. These systems of recurrences are known, at least since the works of A.P. Magnus $[22,23]$ as the Laguerre-Freud equations (see also $[5,20]$ ).

The Laguerre-Freud equations for orthogonal polynomials related to semiclassical weights are often identified with discrete forms of Painlevé equations [31]. Early examples of such identification concern the weight studied by G. Freud [18],

$$
w(x, t)=\exp \left(-x^{4}+t x^{2}\right), \quad x \in \mathbb{R},
$$

where $t$ is a parameter, and the case $t=0$ in (2), studied by J. Shohat in [29]. Here, the Laguerre Freud equations are $4 \gamma_{n}\left(\gamma_{n-1}+\gamma_{n}+\gamma_{n+1}-\frac{t}{2}\right)=$ $n, n=1,2, \ldots$, which are a form of dPI (see $[2,17]$ ). Many other examples of discrete Painlevé equations for the recurrence relation coefficients of orthogonal polynomials have been studied (see [6, 14, 16, 24]). Applications of Laguerre-Freud equations to the study of asymptotics for the orthogonal polynomials, properties of zeroes, estimates for derivatives, inequalities, etc, can be found in a vast list of references (see, amongst many others, [2, 11, 32] and its lists of references).

In this paper we shall consider extensions of semi-classical orthogonal polynomials. We will take the family of Laguerre-Hahn orthogonal polynomials 
$[13,21,26,33]$, that is, the sequences of orthogonal polynomials whose Stieltjes function satisfies a Riccati type differential equation with polynomial coefficients,

$$
A S^{\prime}=B S^{2}+C S+D, \quad A \neq 0,
$$

where $A, B, C, D$ are co-prime. On a general setting, $S$ is the formal moment generating function, defined through the asymptotic expansion

$$
S(x)=\sum_{n=0}^{+\infty} u_{n} x^{-n-1},
$$

given the moments $\left(u_{n}\right)_{n>0}$ of the orthogonality measure. Here, we take, without loss of generality, the normalized sequence of moments, that is, $u_{0}=1$ [33]. The Laguerre-Hahn families of orthogonal polynomials include, as special cases, the semi-classical orthogonal polynomials as well as their standard modifications $[12,33]$. The semi-classical case appears whenever $B \equiv 0$ in (3).

The study of Laguerre-Freud equations for Laguerre-Hahn orthogonal polynomials has been done for several instances of the polynomials $A, B, C, D$ in (3) (see $[1,7,14,15,28]$ ). In this paper we focus on the symmetric class two, that is, we take $s=2$ in [1, Prop. 3.1], thus, we consider the symmetric case under the bounds

$$
\max \{\operatorname{deg}(C)-1, \max \{\operatorname{deg}(A), \operatorname{deg}(B)\}-2\}=2
$$

in equation (3). In the symmetric case, the moments in (4) satisfy $u_{2 n-1}=$ $0, n \geq 1$. We shall take sequences of monic orthogonal polynomials, $P_{n}(x)=$ $x^{n}+$ lower degree terms, $n \geq 0$, satisfying (1), and we denote them by SMOP. We also consider the sequence of associated polynomials of the first kind, $\left\{P_{n}^{(1)}\right\}_{n \geq 0}$, satisfying a three-term recurrence relation

$$
P_{n}^{(1)}(x)=x P_{n-1}^{(1)}(x)-\gamma_{n} P_{n-2}^{(1)}(x), \quad n=1,2, \ldots
$$

with $P_{-1}^{(1)}(x)=0, P_{0}^{(1)}(x)=1$. Combining the recurrence relations (1) and (6) in the matrix form, yields

$$
Y_{n}=\mathcal{A}_{n} Y_{n-1}, \quad Y_{n}=\left[\begin{array}{cc}
P_{n+1} & P_{n}^{(1)} \\
P_{n} & P_{n-1}^{(1)}
\end{array}\right], \quad \mathcal{A}_{n}=\left[\begin{array}{cc}
x & -\gamma_{n} \\
1 & 0
\end{array}\right], \quad n \geq 1,
$$

with initial conditions

$$
Y_{0}=\left[\begin{array}{ll}
x & 1 \\
1 & 0
\end{array}\right]
$$


With the matrices $Y_{n}$ defined above, the SMOP related to the Riccati equation (3), $A S^{\prime}=B S^{2}+C S+D$, satisfy differential systems that can be put into the matrix form as the matrix Sylvester equation [8],

$$
A Y_{n}^{\prime}=\mathcal{B}_{n} Y_{n}-Y_{n} \mathcal{C}, \quad n \geq 0,
$$

where $\mathcal{C}=\left[\begin{array}{cc}C / 2 & -D \\ B & -C / 2\end{array}\right]$ and the matrices $\mathcal{B}_{n}$ are defined in terms of polynomials $l_{n}, \Theta_{n}$ of uniformly bounded degrees,

$\mathcal{B}_{n}=\left[\begin{array}{cc}l_{n} & \Theta_{n} \\ -\Theta_{n-1} / \gamma_{n} & l_{n-1}+x \Theta_{n-1} / \gamma_{n}\end{array}\right], n \geq 1, \mathcal{B}_{0}=\left[\begin{array}{cc}l_{0} & \Theta_{0} \\ -\Theta_{-1} & l_{-1}+x \Theta_{-1}\end{array}\right]$,

where, in the account of (7), the following initial conditions hold:

$$
\begin{gathered}
\Theta_{-1}=D, \Theta_{0}=A+x\left(C / 2-l_{0}\right)+B, \\
l_{-1}=C / 2, l_{0}=-C / 2-x D .
\end{gathered}
$$

Furthermore, combining the recurrence relation (1) with the differential system (8) yields the Lax pair

$$
\left\{\begin{array}{l}
Y_{n}=\mathcal{A}_{n} Y_{n-1}, \\
A Y_{n}^{\prime}=\mathcal{B}_{n} Y_{n}-Y_{n} \mathcal{C},
\end{array}\right.
$$

and, consequently, we get the compatibility conditions for the matrices $\mathcal{A}_{n}$,

$$
A \mathcal{A}_{n}^{\prime}=\mathcal{B}_{n} \mathcal{A}_{n}-\mathcal{A}_{n} \mathcal{B}_{n-1}, \quad n \geq 1 .
$$

In turn, equations (12) yield the following identities:

$$
\begin{gathered}
\operatorname{tr} \mathcal{B}_{n}=0, \quad n \geq 0, \\
\operatorname{det} \mathcal{B}_{n}=\operatorname{det} \mathcal{B}_{0}+A \sum_{k=1}^{n} \frac{\Theta_{k-1}}{\gamma_{k}}, \quad n \geq 1,
\end{gathered}
$$

where $\operatorname{det} \mathcal{B}_{0}=D(A+B)-(C / 2)^{2}$.

Let us emphasize that the Sylvester equations (8) can be regarded as an extension of the differential systems for semi-classical orthogonal polynomials in [24, Eq. 17]. Equation (14) is the analogue of A.P. Magnus' summation formula [24, Eq. 20].

The differential systems enclosed by (12), together with the identities (13) and (14), will be our main tools to deduce difference equations for the recurrence coefficients $\gamma_{n}$ as well as for other relevant coefficients of the orthogonal 
polynomials related to the Riccati equation (3). Let us also emphasize that, in the semi-classical case $(B \equiv 0$ in $(3))$, other methods to get the difference equations for the recurrence coefficients are available, for instance, the Ladder Operator technique [4, Section 4], and the Riemann-Hilbert method $[6,32]$.

The reminder of the paper is organized as follows. In Section 2 we deduce recurrences involving the coefficients $\gamma_{n}$. We stress the results in Theorems 4 and 5 , where we deduce discrete Painlevé equations when $\operatorname{deg}(A)=0$ and $\operatorname{deg}(A)=2$; it is deduced a d-PI and d-PII, respectively. In section 3 we give applications of the previous results, we show examples related to semi-classical as well as to non semi-classical orthogonal polynomials.

\section{The symmetric Laguerre-Hahn class two}

2.1. Fundamental quantities. In this section we derive fundamental quantities to be used throughout the paper.

Henceforth we will use the following convention: if $i>j$, then $\sum_{i}^{j} \cdot=0$.

Taking into account the recurrence relations (1) and (6), we obtain the expansions given in the following lemma.

Lemma 1. Let $\left\{P_{n}\right\}_{n \geq 0}$ be a symmetric SMOP. The following expansions hold, for all $n \geq 1$ :

$$
\begin{aligned}
P_{n+1}(x) & =x^{n+1}+\mathbf{p}_{1}(n+1) x^{n-1}+p_{n+1, n-3} x^{n-3}+\ldots, \\
P_{n}^{(1)}(x) & =x^{n}+\nu_{n} x^{n-2}+p_{n, n-4}^{(1)} x^{n-4}+\ldots,
\end{aligned}
$$

with

$$
\mathbf{p}_{1}(n+1)=-\sum_{k=1}^{n} \gamma_{k}, \quad \mathbf{p}_{1}(1)=0, \quad \nu_{n}=-\sum_{k=2}^{n} \gamma_{k}
$$

and

$$
\begin{aligned}
p_{n+1, n-3} & =\gamma_{1} \gamma_{3}+\sum_{k=4}^{n} \gamma_{k}\left(\gamma_{1}+\cdots+\gamma_{k-2}\right), \quad n \geq 3, \\
p_{n, n-4}^{(1)} & =\gamma_{2} \gamma_{4}+\sum_{k=5}^{n} \gamma_{k}\left(\gamma_{2}+\cdots+\gamma_{k-2}\right), \quad n \geq 4 .
\end{aligned}
$$

Also, the following relation holds:

$$
p_{n+1, n-3}=-\gamma_{1}\left(\gamma_{1}+\gamma_{2}\right)-\gamma_{1} \mathbf{p}_{1}(n+1)+p_{n, n-4}^{(1)} .
$$


Recall that throughout the paper we are considering the Riccati equation $A S^{\prime}=B S^{2}+C S+D$ in the symmetric setting with the sequence of moments $\left(u_{n}\right)$ normalized, that is, $u_{2 n-1}=0, \geq 1, u_{0}=1$. Let the bounds (5) hold. According to [1, Prop. 3.1], $A, B$ must be even, and $C$ odd. Let us write

$$
\begin{array}{cc}
A(x)=a_{4} x^{4}+a_{2} x^{2}+a_{0}, & B(x)=b_{4} x^{4}+b_{2} x^{2}+b_{0}, \\
C(x)=c_{3} x^{3}+c_{1} x, & D(x)=d_{2} x^{2}+d_{0} .
\end{array}
$$

The polynomial $D$ is defined in terms of $A, B, C$ as follows:

$$
d_{2}=-a_{4}-b_{4}-c_{3}, \quad d_{0}=-a_{2}-b_{2}-c_{1}-\gamma_{1}\left(3 a_{4}+2 b 4+c_{3}\right) .
$$

The data from (23) is obtained by equating coefficients of $x^{n+3}$ and $x^{n+1}$, respectively, from the equation enclosed in position $(1,2)$ from (8),

$$
A\left(P_{n}^{(1)}\right)^{\prime}=\left(l_{n}+C / 2\right) P_{n}^{(1)}+\Theta_{n} P_{n-1}^{(1)}+D P_{n+1} .
$$

Indeed, $d_{2}$ is determined once we use $l_{n, 3}$ given by $(25)$ and $d_{0}$ is determined once we use $l_{n, 1}$ given by (26).

Furthermore, the parameter $\gamma_{1}$ is related to the moment of order two. Indeed, the coefficient of $x^{0}$ in (3) gives us, in the account of the asymptotic expansion (4) with $u_{0}=1, d_{0}=-a_{2}-b_{2}-c_{1}-\left(3 a_{4}+2 b_{4}+c_{3}\right) u_{2}$. This, combined with $d_{0}$ given in (23), yields $\gamma_{1}=u_{2}$.

Lemma 2. Let $S$ be a Stieltjes function satisfying $A S^{\prime}=B S^{2}+C S+D$ with $A, B, C, D$ given as in (21)-(22), with $D$ given through (23). Let $\left\{P_{n}\right\}_{n \geq 0}$ be the symmetric $S M O P$ associated with $S$, satisfying the recurrence relation $P_{n+1}(x)=x P_{n}(x)-\gamma_{n} P_{n-1}(x), \quad n=0,1,2, \ldots$ The polynomials $l_{n}, \Theta_{n}$ in (9) are defined by

$$
l_{n}(x)=l_{n, 3} x^{3}+l_{n, 1} x, \quad \Theta_{n}(x)=\Theta_{n, 2} x^{2}+\Theta_{n, 0} .
$$

where, for all $n \geq 1$,

$$
\begin{aligned}
& l_{n, 3}=(n+1) a_{4}+b_{4}+c_{3} / 2, \\
& l_{n, 1}=-2 a_{4} \mathbf{p}_{1}(n+1)+(n+1) a_{2}+\lambda-\Theta_{n, 2}, \\
& \frac{\Theta_{n, 2}}{\gamma_{n+1}}=-\left((2 n+3) a_{4}+\mu\right), \\
& \Theta_{n, 0}=-\gamma_{n} \Theta_{n, 2}-2 a_{2} \mathbf{p}_{1}(n+1)+2 a_{4} \mathbf{p}_{1}^{2}(n+1)-4 a_{4} p_{n+1, n-3} \\
& \quad+(n+1) a_{0}+\tau,
\end{aligned}
$$


where

$$
\lambda=\gamma_{1} b_{4}+b_{2}+c_{1} / 2, \quad \mu=2 b_{4}+c_{3}, \quad \tau=b_{4}\left(\gamma_{1}+\gamma_{2}\right) \gamma_{1}+b_{2} \gamma_{1}+b_{0} .
$$

Alternatively, $\Theta_{n, 0}$ is given, for all $n \geq 1$, by

$$
\frac{\Theta_{n, 0}}{\gamma_{n+1}}=4 a_{4} \mathbf{p}_{1}(n+1)-(2 n+3) a_{2}-2 \lambda-\left((2 n+5) a_{4}+\mu\right)\left(\gamma_{n+2}+\gamma_{n+1}\right) \text {. }
$$

Also, we have the initial conditions

$$
\begin{gathered}
l_{0,3}=a_{4}+b_{4}+\frac{c_{3}}{2}, \quad l_{0,1}=a_{2}+b_{2}+\frac{c_{1}}{2}+\left(3 a_{4}+2 b_{4}+c_{3}\right) \gamma_{1}, \\
\frac{\Theta_{0,2}}{\gamma_{1}}=-3 a_{4}-2 b_{4}-c_{3} \\
\frac{\Theta_{0,0}}{\gamma_{1}}=-3 a_{2}-2 b_{2}-c_{1}-\left(5 a_{4}+c_{3}+3 b_{4}\right) \gamma_{1}-\left(5 a_{4}+\mu\right) \gamma_{2} .
\end{gathered}
$$

Proof: Take the condition enclosed by position $(1,1)$ in $(8)$,

$$
A P_{n+1}^{\prime}=\left(l_{n}-C / 2\right) P_{n+1}+\Theta_{n} P_{n}-B P_{n}^{(1)} .
$$

For all $n \geq 1$, the coefficient of $x^{n+4}$ gives us (25). The coefficient of $x^{n+3}$ gives us

$$
l_{n, 2}=0, \quad n \geq 0 .
$$

For all $n \geq 1$, the coefficient of $x^{n+2}$ gives us (26). The coefficient of $x^{n+1}$ gives us

$$
l_{n, 0}=-\Theta_{n, 1}, \quad n \geq 0 .
$$

For all $n \geq 1$, the coefficient of $x^{n}$ gives us (28).

On the other hand, let us take (13) for $n \geq 1$. It reads

$$
l_{n}(x)+l_{n-1}(x)+x \frac{\Theta_{n-1}(x)}{\gamma_{n}}=0, \quad n \geq 1 .
$$

The coefficient of $x^{2}$ in (37) gives us, in the account of (35),

$$
\frac{\Theta_{n-1,1}}{\gamma_{n}}=0, \quad n \geq 1 \text {. }
$$

Therefore, from (36), we obtain

$$
l_{n, 0}=0, \quad n \geq 0 .
$$

The coefficient of $x^{3}$ in (37) yields $l_{n, 3}+l_{n-1,3}+\frac{\Theta_{n-1,2}}{\gamma_{n}}=0$, from which we get $(27)$. 
The alternative form for $\Theta_{n, 0}$ is obtained from the coefficient of $x$ in (37), $l_{n, 1}+l_{n-1,1}+\frac{\Theta_{n-1,0}}{\gamma_{n}}=0$, from which we get (30).

To obtain (31), we use $l_{0}=-C / 2-x D$ (cf. (11)), thus we get

$$
l_{0,3}=-\frac{c_{3}}{2}-d_{2}, \quad l_{0,1}=-\frac{c_{1}}{2}-d_{0},
$$

which gives the required identities.

To get (32) and (33) we use (37) with $n=1$, hence,

$$
\frac{\Theta_{0,2}}{\gamma_{1}}=-l_{1,3}-l_{0,3}, \quad \frac{\Theta_{0,0}}{\gamma_{1}}=-l_{1,1}-l_{0,1},
$$

which gives the required identities.

Lemma 3. Let the previous notations hold. The coefficients $\gamma_{2}$ and $\gamma_{3}$ are defined in terms of $\gamma_{1}$ through the following equations:

$$
\begin{aligned}
& \frac{a_{0}+b_{0}}{\gamma_{1}}=-3 a_{2}-2 b_{2}-c_{1}-\left(5 a_{4}+c_{3}+3 b_{4}\right) \gamma_{1}-\left(5 a_{4}+\mu\right) \gamma_{2}, \\
& \left(\left(-4 a_{4}-2 b_{4}\right) \gamma_{1}-5 a_{2}-2 b_{2}-c_{1}-\left(7 a_{4}+\mu\right)\left(\gamma_{3}+\gamma_{2}\right)\right) \gamma_{2}=a_{0}+\gamma_{1} d_{0} .
\end{aligned}
$$

Proof: From (10) we get $\Theta_{0,0}=a_{0}+b_{0}$, which we combine with (33), thus getting (40).

Taking $n=1$ in equation (24) and equating the independent term, we get $a_{0}=\Theta_{1,0}-d_{0} \gamma_{1}$. Thus, we get (41).

Remark . Alternatively, (40) can be obtained as follows: the coefficient of $x^{n}$ in (34) and the coefficient of $x^{n-1}$ in (24) give us, respectively, after computations where we use (20),

$$
\begin{aligned}
\gamma_{n} \Theta_{n, 2}+\Theta_{n, 0}=-2 a_{2} \mathbf{p}_{1}(n+1)+ & 2 a_{4} \mathbf{p}_{1}^{2}(n+1) \\
& -4 a_{4} p_{n+1, n-3}+(n+1) a_{0}+\tau \\
\gamma_{n} \Theta_{n, 2}+\Theta_{n, 0}=-2 a_{2} \mathbf{p}_{1}(n+1)+ & 2 a_{4} \mathbf{p}_{1}^{2}(n+1) \\
- & 4 a_{4} p_{n+1, n-3}+n a_{0}+\tilde{\tau}
\end{aligned}
$$

with

$$
\begin{aligned}
& \tau=b_{4}\left(\gamma_{1}+\gamma_{2}\right) \gamma_{1}+b_{2} \gamma_{1}+b_{0}, \\
& \tilde{\tau}=-\left(3 a_{2}+b_{2}+c_{1}+\left(5 a_{4}+c_{3}+2 b_{4}\right) \gamma_{1}+\left(5 a_{4}+c_{3}+b_{4}\right) \gamma_{2}\right) \gamma_{1} .
\end{aligned}
$$

Equating (42) with (43), we get $a_{0}+\tau=\tilde{\tau}$, thus obtaining (40). 


\subsection{Difference equations for $\gamma_{n}$ and $\mathbf{p}_{1}(n)$.}

Theorem 1. Let $S$ be a Stieltjes function satisfying $A S^{\prime}=B S^{2}+C S+D$ with $A, B, C, D$ given as in (21)-(22), with $D$ given through (23). Let $\left\{P_{n}\right\}_{n \geq 0}$ be the symmetric $S M O P$ associated with $S$, satisfying the recurrence relation (1), $P_{n+1}(x)=x P_{n}(x)-\gamma_{n} P_{n-1}(x), \quad n=0,1,2, \ldots$ Let the previous notations hold. The recurrence coefficients $\gamma_{n}$ satisfy the following equation:

$$
\gamma_{n+1} T_{n+1}=\gamma_{n} T_{n-1}+a_{0}, \quad n \geq 2,
$$

with

$$
T_{n}=4 a_{4} \mathbf{p}_{1}(n)-(2 n+1) a_{2}-2 \lambda-\left((2 n+3) a_{4}+\mu\right)\left(\gamma_{n+1}+\gamma_{n}\right), \quad n \geq 2,
$$

and the initial condition $T_{1}=\frac{\Theta_{0,0}}{\gamma_{1}}$. The quantities $\lambda, \mu$ are given in (29).

Proof: The independent term of the equation enclosed in position $(1,1)$ of (12), that is,

$$
A=x\left(l_{n}-l_{n-1}\right)+\Theta_{n}-\gamma_{n} \frac{\Theta_{n-2}}{\gamma_{n-1}}
$$

gives us

$$
a_{0}=\Theta_{n, 0}-\gamma_{n} \frac{\Theta_{n-2,0}}{\gamma_{n-1}}
$$

Hence, we have

$$
a_{0}=\gamma_{n+1} T_{n+1}-\gamma_{n} T_{n-1},
$$

with the identification $T_{n}=\frac{\Theta_{n-1,0}}{\gamma_{n}}$, with $\frac{\Theta_{n-1,0}}{\gamma_{n}}$ given by (30).

Corollary 1. Take $a_{0}=0$ in Theorem 1. The quantities $T_{n}$ satisfy

$$
T_{n+1} T_{n}=\frac{\gamma_{2} T_{2} T_{1}}{\gamma_{n+1}}, \quad n \geq 2 .
$$

Proof: If $a_{0}=0$, then from (44) we get

$$
\gamma_{n+1} T_{n+1} T_{n}=\gamma_{n} T_{n} T_{n-1}, \quad n \geq 2 .
$$

Iteration gives us

$$
\gamma_{n+1} T_{n+1} T_{n}=\gamma_{2} T_{2} T_{1}
$$

Thus, we get (47). 
Corollary 2. The recurrence coefficients $\gamma_{n}$ may be determined recursively through the following equation:

$$
\begin{aligned}
& \gamma_{n+2}=\frac{-a_{0}+E_{n} \gamma_{n+1}-F_{n} \gamma_{n}}{\left((2 n+5) a_{4}+\mu\right) \gamma_{n+1}}, \quad n \geq 2, \\
& \gamma_{3}=-\gamma_{2}-\frac{\left(a_{0}+\gamma_{1} d_{0}\right) / \gamma_{2}+\left(4 a_{4}+2 b_{4}\right) \gamma_{1}+5 a_{2}+2 b_{2}+c_{1}}{7 a_{4}+\mu}, \\
& \gamma_{2}=-\frac{\left(a_{0}+b_{0}\right) / \gamma_{1}+\left(5 a_{4}+c_{3}+3 b_{4}\right) \gamma_{1}+3 a_{2}+2 b_{2}+c_{1}}{5 a_{4}+\mu},
\end{aligned}
$$

with

$$
\begin{gathered}
E_{n}=4 a_{4} \mathbf{p}_{1}(n+1)-(2 n+3) a_{2}-2 \lambda-\left((2 n+5) a_{4}+\mu\right) \gamma_{n+1}, \\
F_{n}=4 a_{4} \mathbf{p}_{1}(n-1)-(2 n-1) a_{2}-2 \lambda-\left((2 n+1) a_{4}+\mu\right)\left(\gamma_{n}+\gamma_{n-1}\right) .
\end{gathered}
$$

Proof: Solving (44) for $\gamma_{n+2}$ gives us (48). The coefficients $\gamma_{2}$ and $\gamma_{3}$ are given from (40) and (41).

Corollary 3. If $a_{4} \neq 0$, the coefficient $\mathbf{p}_{1}(n)$ is determined in terms of $\gamma_{n+2}, \gamma_{n+1}, \gamma_{n}, \gamma_{n-1}$ through the following equation:

$$
\mathbf{p}_{1}(n)=\frac{a_{0}+G_{n} \gamma_{n+1}-H_{n} \gamma_{n}}{4 a_{4}\left(\gamma_{n+1}-\gamma_{n}\right)}, \quad n \geq 2,
$$

with

$$
\begin{gathered}
G_{n}=4 a_{4} \gamma_{n}+(2 n+3) a_{2}+2 \lambda+\left((2 n+5) a_{4}+\mu\right)\left(\gamma_{n+2}+\gamma_{n+1}\right), \\
H_{n}=-4 a_{4} \gamma_{n-1}+(2 n-1) a_{2}+2 \lambda+\left((2 n+1) a_{4}+\mu\right)\left(\gamma_{n}+\gamma_{n-1}\right) .
\end{gathered}
$$

Furthermore, we have:

$$
\mathbf{p}_{1}(n-1)=\frac{a_{0}+\tilde{G}_{n} \gamma_{n+1}-\tilde{H}_{n} \gamma_{n}}{4 a_{4}\left(\gamma_{n+1}-\gamma_{n}\right)}, \quad n \geq 2,
$$

with

$$
\tilde{G}_{n}=G_{n}+4 a_{4} \gamma_{n-1}, \quad \tilde{H}_{n}=H_{n}+4 a_{4} \gamma_{n-1} .
$$

Proof: Using $\mathbf{p}_{1}(n+1)=\mathbf{p}_{1}(n)-\gamma_{n}$ and $\mathbf{p}_{1}(n-1)=\mathbf{p}_{1}(n)+\gamma_{n-1}$ in $(44)$ and solving for $\mathbf{p}_{1}(n)$ we get (51).

Using $\mathbf{p}_{1}(n+1)=\mathbf{p}_{1}(n-1)-\gamma_{n-1}-\gamma_{n}$ in $(44)$ and solving for $\mathbf{p}_{1}(n-1)$ we get (54).

Doing the shift $n \rightarrow n-1$ in (51) and equating to (54) we get a fourth order difference equation for $\gamma_{n}$, as stated in the following corollary. 
Corollary 4. The recurrence coefficients $\gamma_{n}$ satisfy the fourth order difference equation

$$
\gamma_{n+2}=\frac{\mathcal{F}\left(\gamma_{n+1}, \gamma_{n}, \gamma_{n-1}, \gamma_{n-2}\right)}{\left((2 n+5) a_{4}+\mu\right) \gamma_{n+1}\left(\gamma_{n-1}-\gamma_{n}\right)}, \quad n \geq 2
$$

with

$$
\begin{aligned}
& \mathcal{F}\left(\gamma_{n+1}, \gamma_{n}, \gamma_{n-1}, \gamma_{n-2}\right)=-a_{0}\left(\gamma_{n+1}-2 \gamma_{n}+\gamma_{n-1}\right) \\
& \quad+\gamma_{n+1} \gamma_{n}\left[2 a_{4} \gamma_{n+1}-\left((2 n-1) a_{4}+\mu\right) \gamma_{n}+2 a_{2}\right] \\
& +\gamma_{n+1} \gamma_{n-1}\left[-\left((2 n+5) a_{4}+\mu\right) \gamma_{n+1}-4 a_{4} \gamma_{n}+\left((2 n-5) a_{4}+\mu\right)\left(\gamma_{n-1}+\gamma_{n-2}\right)-6 a_{2}\right] \\
& \quad+\gamma_{n}^{2}\left[\left((2 n+3) a_{4}+\mu\right) \gamma_{n+1}+2 a_{4} \gamma_{n}-\left((2 n-3) a_{4}+\mu\right) \gamma_{n-1}+2 a_{2}\right] \\
& \quad+\gamma_{n} \gamma_{n-1}\left[\left((2 n+1) a_{4}+\mu\right) \gamma_{n}+2 a_{4} \gamma_{n-1}-\left((2 n-5) a_{4}+\mu\right) \gamma_{n-2}+2 a_{2}\right] .
\end{aligned}
$$

Theorem 2. Let $S$ be a Stieltjes function satisfying $A S^{\prime}=B S^{2}+C S+D$ with $A, B, C, D$ given as in (21)-(22), with $D$ given through (23). Let $\left\{P_{n}\right\}_{n \geq 0}$ be the symmetric SMOP associated with $S$, satisfying the recurrence relation (1), $P_{n+1}(x)=x P_{n}(x)-\gamma_{n} P_{n-1}(x), \quad n=0,1,2, \ldots$ Let the previous notations hold, as well as

$$
\mu_{n}=(2 n+1) a_{4}+\mu, \quad \lambda_{n}=(2 n+1) a_{2}+2 \lambda,
$$

where $\lambda$ and $\mu$ are given in (29). The coefficients $\mathbf{p}_{1}(n)$ given in (17) satisfy the following quadratic equations:

$$
\begin{aligned}
& 4 a_{4}^{3} \mathbf{p}_{1}^{2}(n+1)+B_{n+1} \mathbf{p}_{1}(n+1)+C_{n+1}=0, \\
& 16 a_{4}^{3} \gamma_{n+1} \mathbf{p}_{1}^{2}(n+1)+\tilde{B}_{n+1} \mathbf{p}_{1}(n+1)+\tilde{C}_{n+1}=0,
\end{aligned}
$$

where

$$
\begin{gathered}
B_{n+1}=4 a_{4}\left[a_{2}\left(\frac{c_{3}}{2}+b_{4}\right)-a_{4} \lambda+a_{4} \mu_{n} \gamma_{n+1}\right] \\
C_{n+1}=-a_{4}\left[\mu_{n} \mu_{n+2} \gamma_{n+2}+\mu_{n} \mu_{n+2} \gamma_{n+1}+\mu_{n+1} \mu_{n-1} \gamma_{n}\right. \\
\left.\quad+\mu_{n} \lambda_{n+1}\right] \gamma_{n+1}+\tau_{n}
\end{gathered}
$$


where

$$
\begin{gathered}
\tau_{n}=\left(a_{4} \xi_{0,2}-a_{2} \xi_{0,4}\right)+\left(a_{0} a_{4}-a_{2}^{2}\right)\left(\frac{\Theta_{0,2}}{\gamma_{1}}-(n-1)\left((n+3) a_{4}+\mu\right)\right) \\
-\left((n+1) a_{2}+\lambda\right)\left(a_{4}\left((n+1) a_{2}-\lambda\right)+a_{2} \mu\right), \\
\tilde{\tau}_{n}=2 a_{0} l_{n, 3}\left((n+1) a_{2}+\lambda\right)+a_{0} \xi_{0,4}-a_{4} \xi_{0,0}+a_{0} a_{2}\left(\frac{\Theta_{0,2}}{\gamma_{1}}-(n-1)\left((n+3) a_{4}+\mu\right)\right) .
\end{gathered}
$$

Here, $\xi_{0, j}$ denotes the coefficient of $x^{j}$ in $\operatorname{det} \mathcal{B}_{0}$.

Proof: Take the coefficients of $x^{4}, x^{2}$ and $x^{0}$ in (14), i.e., in equation

$$
-l_{n}^{2}(x)+\Theta_{n}(x) \frac{\Theta_{n-1}(x)}{\gamma_{n}}=\operatorname{det} \mathcal{B}_{0}+A \sum_{k=1}^{n} \frac{\Theta_{k-1}}{\gamma_{k}}, \quad n \geq 1 \text {. }
$$

We get, respectively,

$$
\begin{aligned}
& -2 l_{n, 1} l_{n, 3}+\Theta_{n, 2} \frac{\Theta_{n-1,2}}{\gamma_{n}}=\xi_{0,4}+a_{4} \sum_{k=1}^{n} \frac{\Theta_{k-1,0}}{\gamma_{k}}+a_{2} \sum_{k=1}^{n} \frac{\Theta_{k-1,2}}{\gamma_{k}} \\
& -l_{n, 1}^{2}+\Theta_{n, 2} \frac{\Theta_{n-1,0}}{\gamma_{n}}+\Theta_{n, 0} \frac{\Theta_{n-1,2}}{\gamma_{n}}=\xi_{0,2}+a_{2} \sum_{k=1}^{n} \frac{\Theta_{k-1,0}}{\gamma_{k}}+a_{0} \sum_{k=1}^{n} \frac{\Theta_{k-1,2}}{\gamma_{k}}(, 64) \\
& \Theta_{n, 0} \frac{\Theta_{n-1,0}}{\gamma_{n}}=\xi_{0,0}+a_{0} \sum_{k=1}^{n} \frac{\Theta_{k-1,0}}{\gamma_{k}} .
\end{aligned}
$$

Eliminating $\sum_{k=1}^{n} \frac{\Theta_{k-1,0}}{\gamma_{k}}$ between (63) and (64), and using the data from Lemma 2, we get, after simplifications, (56). Eliminating $\sum_{k=1}^{n} \frac{\Theta_{k-1,0}}{\gamma_{k}}$ between (63) and (65), and using the data from Lemma 2, we get, after simplifications, (57).

Theorem 3. Let the notations and conditions of Theorem 2 hold. The recurrence coefficients $\gamma_{n}$ satisfy the following second order difference equation:

$$
\sum_{p=0}^{3} \sum_{q=0}^{6} \sum_{r=0}^{3} c_{p, q, r} \gamma_{n}^{p} \gamma_{n+1}^{q} \gamma_{n+2}^{r}=0
$$

Proof: Eliminating the quadratic term between (56) and (57), we get

$$
\mathbf{p}_{1}(n+1)=\frac{\tilde{C}_{n+1}-4 \gamma_{n+1} C_{n+1}}{4 \gamma_{n+1} B_{n+1}-\tilde{B}_{n+1}}
$$


from which we obtain, by substitution into (56), the equation

$$
\begin{aligned}
4 a_{4}^{3}\left(\tilde{C}_{n+1}-4 \gamma_{n+1} C_{n+1}\right)^{2}+B_{n+1}\left(\tilde{C}_{n+1}-\right. & \left.4 \gamma_{n+1} C_{n+1}\right)\left(4 \gamma_{n+1} B_{n+1}-\tilde{B}_{n+1}\right) \\
& +C_{n+1}\left(4 \gamma_{n+1} B_{n+1}-\tilde{B}_{n+1}\right)^{2}=0 .
\end{aligned}
$$

Therefore, we obtain (66) with coefficients $c_{p, q, r}$ defined in terms of $\lambda_{n}, \mu_{n}, \tau_{n}$, and $\tilde{\tau}_{n}$.

2.3. Difference equations of the Painlevé type. In this subsection we derive difference equations of the Painlevé type for symmetric Laguerre-Hahn orthogonal polynomials of class two. The fundamental tools are the identities for the trace and determinant given by (13) and (14). Recall these identities reading as (37) and (62), respectively:

$$
\begin{gathered}
l_{n}(x)+l_{n-1}(x)+x \frac{\Theta_{n-1}(x)}{\gamma_{n}}=0, \quad n \geq 0, \\
-l_{n}^{2}(x)+\Theta_{n}(x) \frac{\Theta_{n-1}(x)}{\gamma_{n}}=\operatorname{det} \mathcal{B}_{0}+A \sum_{k=1}^{n} \frac{\Theta_{k-1}}{\gamma_{k}}, \quad n \geq 1 .
\end{gathered}
$$

In what follows we consider the cases $\operatorname{deg}(A)=0$ and $\operatorname{deg}(A)=2$ in the Riccati equation $A S^{\prime}=B S^{2}+C S+D$. Without loss of generality, the polynomial $A$ will be taken as monic.

Theorem 4. Let $S$ be a Stieltjes function satisfying $A S^{\prime}=B S^{2}+C S+D$ with

$A(x)=1, \quad B(x)=b_{4} x^{4}+b_{2} x^{2}+b_{0}, \quad C(x)=c_{3} x^{3}+c_{1} x, \quad D(x)=d_{2} x^{2}+d_{0}$,

with $d_{2}, d_{0}$ given in (23). Let $\left\{P_{n}\right\}_{n \geq 0}$ be the symmetric SMOP associated with $S$, satisfying the recurrence relation $P_{n+1}(x)=x P_{n}(x)-\gamma_{n} P_{n-1}(x), n=$ $0,1,2, \ldots$ The coefficients $\gamma_{n}$ satisfy the discrete Painlevé I equation

$$
\gamma_{n}\left(\mu\left(\gamma_{n-1}+\gamma_{n}+\gamma_{n+1}\right)+2 \lambda\right)=-n-\tau, \quad n \geq 1,
$$

with $\lambda, \mu, \tau$ given in (29),

$$
\lambda=\gamma_{1} b_{4}+b_{2}+c_{1} / 2, \quad \mu=2 b_{4}+c_{3}, \quad \tau=b_{4}\left(\gamma_{1}+\gamma_{2}\right) \gamma_{1}+b_{2} \gamma_{1}+b_{0} .
$$

Proof: From Lemma 2, we have

$$
l_{n, 1}=\lambda-\Theta_{n, 2}, \quad \Theta_{n, 0}=-\gamma_{n} \Theta_{n, 2}+n+1+\tau, \quad \Theta_{n, 2}=-\mu \gamma_{n+1},
$$


with $\lambda, \mu, \tau$ given in (29). Using these equalities into the relation that follows from the linear term in the equation for the trace (37),

$$
l_{n, 1}+l_{n-1,1}+\frac{\Theta_{n-1,0}}{\gamma_{n}}=0,
$$

we obtain (68).

Theorem 5. Let $S$ be a Stieltjes function satisfying $A S^{\prime}=B S^{2}+C S+D$ with

$A(x)=x^{2}+a_{0}, B(x)=b_{4} x^{4}+b_{2} x^{2}+b_{0}, C(x)=c_{3} x^{3}+c_{1} x, D(x)=d_{2} x^{2}+d_{0}$,

with $d_{2}, d_{0}$ given in (23). Let $\left\{P_{n}\right\}_{n \geq 0}$ be the symmetric SMOP associated with $S$, satisfying the recurrence relation $P_{n+1}(x)=x P_{n}(x)-\gamma_{n} P_{n-1}(x), n=$ $0,1,2, \ldots$

If

$$
4\left(a_{0} b_{2}-b_{0}\right) d_{0}=a_{0}^{3} \mu^{2}-4 a_{0}^{2} \mu(1+\lambda)-4 a_{0}\left(d_{2}\left(a_{0}+b_{0}\right)-c_{1}^{2} / 4\right),
$$

where $\lambda=\gamma_{1} b_{4}+b_{2}+c_{1} / 2, \mu=2 b_{4}+c_{3}$, then the expression $x_{n}=n+1+$ $\lambda+\mu \gamma_{n+1}-\frac{\mu a_{0}}{2}$ satisfies the discrete Painlevé II equation

$$
\left(x_{n-1}+x_{n}\right)\left(x_{n}+x_{n+1}\right)=\frac{-4 x_{n}^{2}}{\left(\hat{\lambda} x_{n}+z_{n}\right)}, \quad n \geq 1,
$$

with $\hat{\lambda}=\frac{4}{\mu a_{0}}, z_{n}=2-\frac{4(n+1+\lambda)}{\mu a_{0}}$.

Proof: The independent term in the equation for the determinant (62) gives us

$$
\sum_{k=1}^{n} \frac{\Theta_{k-1,0}}{\gamma_{k}}=\frac{1}{a_{0}}\left(\gamma_{n+1} \frac{\Theta_{n, 0}}{\gamma_{n+1}} \frac{\Theta_{n-1,0}}{\gamma_{n}}-\xi_{0,0}\right) .
$$

Using the equation above as well as $\Theta_{n, 2}=-\mu \gamma_{n+1}, n \geq 0$, into the coefficient of the quadratic term of (62),

$$
-l_{n, 1}^{2}+\Theta_{n, 2} \frac{\Theta_{n-1,0}}{\gamma_{n}}+\Theta_{n, 0} \frac{\Theta_{n-1,2}}{\gamma_{n}}=\xi_{0,2}+a_{2} \sum_{k=1}^{n} \frac{\Theta_{k-1,0}}{\gamma_{k}}+a_{0} \sum_{k=1}^{n} \frac{\Theta_{k-1,2}}{\gamma_{k}},
$$

we obtain, after some computations,

$$
-a_{0} l_{n, 1}^{2}-\kappa_{n}=\mu a_{0} \gamma_{n+1}\left(\frac{\Theta_{n-1,0}}{\gamma_{n}}+\frac{\Theta_{n, 0}}{\gamma_{n+1}}\right)+\gamma_{n+1} \frac{\Theta_{n, 0}}{\gamma_{n+1}} \frac{\Theta_{n-1,0}}{\gamma_{n}},
$$


with $\kappa_{n}=a_{0} \xi_{0,2}-\mu a_{0}^{2} n-\xi_{0,0}$. Thus, we have

$$
\gamma_{n+1}\left(\mu a_{0}\right)^{2}-a_{0} l_{n, 1}^{2}-\kappa_{n}=\gamma_{n+1}\left(\frac{\Theta_{n-1,0}}{\gamma_{n}}+\mu a_{0}\right)\left(\frac{\Theta_{n, 0}}{\gamma_{n+1}}+\mu a_{0}\right) .
$$

Taking into account that

$$
l_{n, 1}=n+1+\lambda+\mu \gamma_{n+1},
$$

the left hand side of (73) is quadratic in $\gamma_{n+1}$. Due to condition (71), the left hand side of (73) factorizes as

$$
-a_{0} \mu^{2}\left(\gamma_{n+1}+\varepsilon_{n}\right)^{2}
$$

with $\varepsilon_{n}=\frac{2(n+1+\lambda)-\mu a_{0}}{2 \mu}$.

Hence, we have

$$
-a_{0} \mu^{2}\left(\gamma_{n+1}+\varepsilon_{n}\right)^{2}=\gamma_{n+1}\left(\frac{\Theta_{n-1,0}}{\gamma_{n}}+\mu a_{0}\right)\left(\frac{\Theta_{n, 0}}{\gamma_{n+1}}+\mu a_{0}\right) .
$$

Now we use the identities

$$
\gamma_{n+1}=\frac{l_{n, 1}-(n+1+\lambda)}{\mu}, \quad-\left(l_{n, 1}+l_{n-1,1}\right)=\frac{\Theta_{n-1,0}}{\gamma_{n}}
$$

(cf.(74) and (69)) into (75), thus obtaining

$$
\begin{aligned}
\left(l_{n, 1}-\frac{\mu a_{0}}{2}\right)^{2}=\frac{\left(l_{n, 1}-(n+1+\lambda)\right)}{-\mu a_{0}} & \left(\left(l_{n+1,1}-\frac{\mu a_{0}}{2}\right)+\left(l_{n, 1}-\frac{\mu a_{0}}{2}\right)\right) \\
& \times\left(\left(l_{n, 1}-\frac{\mu a_{0}}{2}\right)+\left(l_{n-1,1}-\frac{\mu a_{0}}{2}\right)\right) .
\end{aligned}
$$

With the identification $x_{n}=l_{n, 1}-\frac{\mu a_{0}}{2}$, the previous equation is written as

$$
\left(x_{n+1}+x_{n}\right)\left(x_{n}+x_{n-1}\right)\left(\tilde{\lambda} x_{n}+\tilde{z}_{n}\right)=x_{n}^{2},
$$

with $\tilde{\lambda}=-\frac{1}{\mu a_{0}}, \tilde{z}_{n}=\frac{n+1+\lambda-\left(\mu a_{0}\right) / 2}{\mu a_{0}}$. Hence, we get the discrete Painlevé II

$$
\left(x_{n+1}+x_{n}\right)\left(x_{n}+x_{n-1}\right)=\frac{-4 x_{n}^{2}}{\left(\hat{\lambda} x_{n}+z_{n}\right)},
$$

with $\hat{\lambda}=\frac{4}{\mu a_{0}}, z_{n}=2-\frac{4(n+1+\lambda)}{\mu a_{0}}$.

Remark. If no cancellations occur, condition (71) yields conditions on $\gamma_{1}$ (i.e, on the normalized moment of order two) for the Painlevé equation to hold. 


\section{Examples}

A way of generating orthogonal polynomials with respect to a symmetric measure is doing a quadratic transformation from the weights related to the classical orthogonal polynomials (see [9]). Some of such transformations falling into the class two are given in the following examples.

3.1. Example 1. Let us take the modified Freud weight [18, 29],

$$
w(x, t)=\exp \left(-x^{4}+t x^{2}\right), \quad x \in \mathbb{R} .
$$

Here, $t$ is a parameter, which, in some contexts, is interpreted as the time variable (see [24]).

$w$ satisfies the Pearson equation $\frac{1}{w} \frac{d}{d x} w=\frac{C}{A}$, where $A(x)=1, C(x)=$ $-4 x^{3}+2 t x$. Thus, in our previous notations,

$$
a_{4}=a_{2}=0, a_{0}=1, c_{3}=-4, c_{1}=2 t .
$$

We take the Stieltjes function related to $w$, satisfying $A S^{\prime}=C S+D$, where $D(x)=d_{2} x^{2}+d_{0}$, with $d_{2}=-a_{4}-c_{3}, d_{0}=-a_{2}-c_{1}-\left(3 a_{4}+c_{3}\right) \gamma_{1}$. Thus, we have

$$
d_{2}=4, \quad d_{0}=-2 t+4 \gamma_{1},
$$

where $\gamma_{1}$ is the normalized moment of order two,

$$
\gamma_{1}=\frac{\int_{\mathbb{R}} x^{2} \exp \left(-x^{4}+t x^{2}\right) d x}{\int_{\mathbb{R}} \exp \left(-x^{4}+t x^{2}\right) d x} .
$$

In the account of the above data we have, from Lemma $2, \lambda=t, \mu=$ $-4, \tau=0$. From Theorem 4, we have the following d-PI (see [17, 24]),

$$
4 \gamma_{n}\left(\gamma_{n-1}+\gamma_{n}+\gamma_{n+1}-\frac{t}{2}\right)=n, \quad n \geq 1
$$

with initial conditions $\gamma_{0}=0$ and $\gamma_{1}$ given by (78).

Let us note that $n=1$ in (79) is compatible with (40) provided $\gamma_{0}=0$, and $n=2$ in (79) agrees with (41).

3.2. Example 2. Let us take the modified Hermite weight $[3,25]$,

$$
\left.\left.w(x, a)=\exp \left(-x^{2}\right), \quad x \in I(a)=\right]-\infty,-a\right] \cup[a,+\infty[,
$$

where $a$ is some positive real number. 
$w$ satisfies the Pearson equation $\frac{1}{w} \frac{d}{d x} w=\frac{C}{A}$, where $A(x)=x^{2}-a^{2}, C(x)=$ $-2 x^{3}+2 a^{2} x$ (see [3]). Thus, in our previous notations,

$$
a_{4}=0, a_{2}=1, a_{0}=-a^{2}, c_{3}=-2, c_{1}=2 a^{2} .
$$

We take the Stieltjes function related to $w$, satisfying $A S^{\prime}=C S+D$, where $D(x)=d_{2} x^{2}+d_{0}$, with $d_{2}=-a_{4}-c_{3}, d_{0}=-a_{2}-c_{1}-\left(3 a_{4}+c_{3}\right) \gamma_{1}$. Thus, we have

$$
d_{2}=2, \quad d_{0}=-1-2 a^{2}+2 \gamma_{1},
$$

where $\gamma_{1}$ is the normalized moment of order two,

$$
\gamma_{1}=\frac{\int_{I(a)} x^{2} \exp \left(-x^{2}\right) d x}{\int_{I(a)} \exp \left(-x^{2}\right) d x} .
$$

The constants $\lambda$ and $\mu$ in Theorem 5 are given by $\lambda=a^{2}, \mu=-2$. Note that condition (71) holds. Therefore, the expression $x_{n}=n+1-2 \gamma_{n+1}$ satisfies the following d-PII,

$$
\left(x_{n-1}+x_{n}\right)\left(x_{n}+x_{n+1}\right)=\frac{-4 x_{n}^{2}}{\left(\hat{\lambda} x_{n}+z_{n}\right)}, \quad n \geq 1,
$$

with $\hat{\lambda}=\frac{2}{a^{2}}, z_{n}=-\frac{2(n+1)}{a^{2}}$. Indeed, the formula (83) holds for $n=0$, under the initial conditions $x_{-1}=0, x_{0}=1-2 \gamma_{1}$, where $\gamma_{1}$ is given by (82).

3.3. Example 3. Let us take the modified Jacobi weight [4],

$$
\left.w(x, k)=\left(1-x^{2}\right)^{\alpha}\left(1-k^{2} x^{2}\right)^{\beta}, \quad x \in[-1,1], \alpha>-1, \beta \in \mathbb{R}, k^{2} \in\right] 0,1[.
$$

$w$ satisfies the Pearson equation $\frac{1}{w} \frac{d}{d x} w=\frac{C}{A}$, where $A(x)=\left(x^{2}-1\right)\left(x^{2}-\right.$ $\left.1 / k^{2}\right), C(x)=(2 \alpha+2 \beta) x^{3}+\left(-2 \alpha / k^{2}-2 \beta\right) x$. Thus, in our previous notations,

$$
a_{4}=1, a_{2}=-\left(1+1 / k^{2}\right), a_{0}=1 / k^{2}, c_{3}=2 \alpha+2 \beta, c_{1}=-2 \alpha / k^{2}-2 \beta .
$$

We take the Stieltjes function related to $w$, satisfying $A S^{\prime}=C S+D$, where $D(x)=d_{2} x^{2}+d_{0}$, with $d_{2}=-a_{4}-c_{3}, \quad d_{0}=-a_{2}-c_{1}-\left(3 a_{4}+c_{3}\right) \gamma_{1}$. Thus, we have

$$
d_{2}=-1-2 \alpha-2 \beta, \quad d_{0}=\left(1+1 / k^{2}\right)+2 \alpha / k^{2}+2 \beta-(3+2 \alpha+2 \beta) \gamma_{1},
$$


where $\gamma_{1}$ is the normalized moment of order two,

$$
\gamma_{1}=\frac{\int_{-1}^{1} x^{2}\left(1-x^{2}\right)^{\alpha}\left(1-k^{2} x^{2}\right)^{\beta} d x}{\int_{-1}^{1}\left(1-x^{2}\right)^{\alpha}\left(1-k^{2} x^{2}\right)^{\beta} d x} .
$$

The coefficients $\gamma_{n}$ of the SMOP related to (84) are governed by the difference equations described in Theorems 1 and 3 , with $b_{4}=b_{2}=b_{0}$ in all formulae.

Remark. The difference equations given in Theorems 1 and 3 with $B \equiv 0$ were also derived in [4], using the ladder operator technique.

\subsection{Further examples - non semi-classical orthogonal polynomi-}

als. Let us now look at some examples of orthogonal polynomials not semiclassical whose results from Section 2 apply.

Let us take the Stieltjes function related to the previous examples, satisfying the differential equation $A S^{\prime}=C S+D$. We now consider the Stieltjes function $S^{(1)}[33]$

$$
\gamma_{1} S^{(1)}(x)=-\frac{1}{S(x)}+x .
$$

$S^{(1)}$ is the Stieltjes function related to the associated polynomials of the first kind, $\left\{P_{n}^{(1)}\right\}_{n \geq 0}$. As $S$ satisfies the linear differential equation $A S^{\prime}=C S+D$, then $S^{(1)}$ satisfies the Riccati equation

$$
A_{1}\left(S^{(1)}\right)^{\prime}=B_{1}\left(S^{(1)}\right)^{2}+C_{1} S^{(1)}+D_{1}
$$

with

$$
A_{1}=A, B_{1}=\gamma_{1} D, C_{1}=-(C+2 x D), D_{1}=\left(A+x C+x^{2} D+B\right) / \gamma_{1},
$$

Note that $\operatorname{deg}\left(D_{1}\right)=2$. The degrees of the polynomials in (88) satisfy (5), therefore, the sequences of orthogonal polynomials $\left\{P_{n}^{(1)}\right\}_{n \geq 0}$ are LaguerreHahn sequences of class two. The recurrence coefficients of $\left\{P_{n}^{(1)}\right\}_{n \geq 0}$, satisfy the difference equations given in Theorems 1 and 3, as well as in Theorems 4 and 5 .

\section{References}

[1] J. Alaya, P. Maroni P, Symmetric Laguerre-Hahn forms of class $s=1$, Integral Transforms Spec. Funct. 4 (1996), 301-320. 
[2] S.M. Alsulami, P. Nevai, J. Szabados, W. Van Assche, A family of nonlinear difference equations: existence uniqueness and asymptotic behaviour of positive solutions, J. Approx. Theory 193 (2015) 39-55.

[3] S. Belmehdi, A. Ronveaux, Laguerre Freud's equations for the recurrence coefficients of semiclassical orthogonal polynomials, J. Approx. Theory 76 (1994) 351-368.

[4] Estelle L. Basor, Yang Chen, Nazmus S. Haq, Asymptotics of determinants of Hankel matrices via non-linear difference equations, J. Approx. Theory 198 (2015) 63-110.

[5] S. Belmehdi and A. Ronveaux, Laguerre-Freud's equations for the recurrence coefficients of semi-classical orthogonal polynomials, J. Approx. Theory. 76 (1994), 351-368.

[6] P. Bleher, A.R. Its, Semiclassical asymptotics of orthogonal polynomials, Riemann-Hilbert problem, and universality in the matrix model, Ann. of Math. 150 (1999) 185-266.

[7] H. Bouakkaz, P. Maroni, Description des polynômes orthogonaux de Laguerre-Hahn de classe zéro, in: Orthogonal Polynomials and Their Applications (Erice 1990), IMACS Ann. Comput. Appl. Math. 9, Baltzer, Basel, 1991, pp. 189194.

[8] A. Branquinho, A. Paiva, and M.N. Rebocho, Sylvester equations for Laguerre-Hahn orthogonal polynomials on the real line, Appl. Math. Comput. 219 (2013), 9118-9131.

[9] T.S. Chihara, An Introduction to Orthogonal Polynomials. Gordon and Breach, New York; 1978.

[10] P.A. Clarkson, K. Jordaan, A. Kelil, A generalized Freud weight, Stud. Appl. Math. 136 (2016), 288-320.

[11] P.A. Clarkson and K. Jordaan, Properties of generalized Freud polynomials, J. Approx. Theory 225 (2018), 148-175.

[12] J.S. Dehesa, F. Marcellán, and A. Ronveaux, On orthogonal polynomials with perturbed recurrence relations, J. Comput. Appl. Math. 30 (1990), 203-212.

[13] J. Dini, Sur les formes linéaires et les polynômes orthogonaux de Laguerre-Hahn [doctoral dissertation]. Paris (France): University Pierre et Marie Curie; 1988.

[14] G. Filipuk, M.N. Rebocho, Discrete Painlevé equations for recurrence coefficients of LaguerreHahn orthogonal polynomials of class one, Integral Transforms Spec. Funct. 27 (2016), 548-565.

[15] G. Filipuk, M.N. Rebocho, Laguerre-Hahn orthogonal polynomials of class one: classification and discrete Painlevé equations, submitted.

[16] G. Filipuk, W. Van Assche, L. Zhang, The recurrence coefficients of semiclassical laguerre polynomials and the fourth Painlevé equation, J. Phys. A 45 (2012) 205201.

[17] A.S. Fokas, A. Its, and A.V. Kitaev, Discrete Painlevé equations and their appearance in quantum gravity, Commun. Math. Phys. 142 (1991), 313-44.

[18] G. Freud, On the coefficients in the recursion formulae of orthogonal polynomials. Proc. Roy. Irish Acad. Sect. A 76(1) (1976), 1-6.

[19] E. Hendriksen, H. van Rossum, Semiclassical orthogonal polynomials, in: C. Brezinski, A. Draux, A.P. Magnus, P. Maroni, A. Ronveaux (Eds.), Orthogonal Polynomials and Applications, in: Lect. Notes Math., vol. 1171, Springer- Verlag, Berlin, 1985, pp. 354-361.

[20] E. Laguerre, Sur la réduction en fractions continues d'une fraction qui satisfait à une équation différentialle linéaire du premier ordre dont les coefficients sont rationnels. J. Math. Pures Appl. (4) 1 (1885), 135-165.

[21] A.P. Magnus, Riccati acceleration of the Jacobi continued fractions and Laguerre-Hahn polynomials. In: Werner H, Bunger HT, editors. Padé Approximation and its Applications. Lect. Notes in Math. 1071, Berlin: Springer Verlag; 1984. p. 213-230.

[22] A.P. Magnus. A proof of Freud's conjecture about the orthogonal polynomials related to $|x|^{\rho} \exp \left(-x^{2 m}\right)$, for integer $m$. In Orthogonal Polynomials and applications (Bar-le-Duc, 1984), vol. 1171 of Lecture Notes in Math., pp. 362-372. Springer, Berlin (1985). 
[23] A.P. Magnus. On Freud's equations for exponential weights, J. Approx. Theory 46(1) (1986), 65-99.

[24] A.P. Magnus, Painlevé-type differential equations for the recurrence coefficients of semiclassical orthogonal polynomials, J. Comput. Appl. Math. 57 (1995), 215-237.

[25] Man Cao, Yang Chen, James Griffin, Continuous and Discrete Painlevé Equations Arising from the Gap Probability Distribution of the Finite $n$ Gaussian Unitary Ensembles, J. Stat. Phys. 157 (2014), 363-375.

[26] P. Maroni, Une théorie algébrique des polynômes orthogonaux. Application aux polynômes orthogonaux semi-classiques. In: Brezinski C, Gori L, Ronveaux A, editors. Orthogonal polynomials and their applications. Baltzer, Basel: IMACS Ann. Comput. Appl. Math. 9; 1991. p. $95-130$.

[27] A. Ronveaux, W. Van Assche, Upward extension of the Jacobi matrix for orthogonal polynomials, J. Approx. Theory 86 (1996) 335-357.

[28] M. Sghaier, J. Alaya, Semiclassical forms of class s $=2$ : the symmetric case, when $\Phi(0)=0$. Methods Appl. Anal. 13 (2006) 387-410.

[29] J. Shohat, A differential equation for orthogonal polynomials, Duke Math. J. 5 (1939) 401-417.

[30] G. Szegö, Orthogonal polynomials. 4th ed. Providence Rhode Island: Amer Math Soc Colloq Publ.; 1975.

[31] W. Van Assche, Discrete Painlevé equations for recurrence coefficients of orthogonal polynomials. In: Elaydi S, Cushing J, Lasser R, Ruffing A, Papageorgiou V, Van Assche W, editors. Difference equations, special functions and orthogonal polynomials. Hackensack, NJ: World Scientific; 2007. pp. 687-725.

[32] R. Wong, L. Zhang, Global asymptotics of orthogonal polynomials associated with $|x|^{2 \alpha} e^{Q(x)}$, J. Approx. Theory 162 (2010) 723-765.

[33] A. Zhedanov, Rational spectral transformations and orthogonal polynomials, J. Comput. Appl. Math. 85 (1997), 67-86.

G. FILIPUK

Faculty of Mathematics, Informatics and Mechanics, University of Warsaw, Banacha 2, WARSAW, 02-097, POLAND.

E-mail address: filipuk@mimuw.edu.pl

M.N. REBOCHO

Departamento de Matemática, Universidade da Beira interior, 6201-001 Covilhã, Portugal; CMUC, Department of Mathematics, University of Coimbra, 3001-501 Coimbra, Portugal.

E-mail address: mneves@ubi.pt 